\title{
Quantum conductance of 4,4-bipyridine molecular junctions: Role of electrode work function and local $d$ band
}

\author{
Rauba, J.M.C.; Strange, Mikkel; Thygesen, Kristian Sommer
}

Published in:

Physical Review B Condensed Matter

Link to article, DOI:

10.1103/PhysRevB.78.165116

Publication date:

2008

Document Version

Publisher's PDF, also known as Version of record

Link back to DTU Orbit

Citation (APA):

Rauba, J. M. C., Strange, M., \& Thygesen, K. S. (2008). Quantum conductance of 4,4-bipyridine molecular junctions: Role of electrode work function and local d band. Physical Review B Condensed Matter, 78(16), 165116. https://doi.org/10.1103/PhysRevB.78.165116

\section{General rights}

Copyright and moral rights for the publications made accessible in the public portal are retained by the authors and/or other copyright owners and it is a condition of accessing publications that users recognise and abide by the legal requirements associated with these rights.

- Users may download and print one copy of any publication from the public portal for the purpose of private study or research.

- You may not further distribute the material or use it for any profit-making activity or commercial gain

- You may freely distribute the URL identifying the publication in the public portal 


\title{
Quantum conductance of 4,4-bipyridine molecular junctions: Role of electrode work function and local $d$ band
}

\author{
J. M. C. Rauba, M. Strange, and K. S. Thygesen \\ Center for Atomic-scale Materials Design (CAMD), Department of Physics, Technical University of Denmark, \\ DK-2800 Kgs. Lyngby, Denmark
}

(Received 17 June 2008; revised manuscript received 9 September 2008; published 17 October 2008)

\begin{abstract}
We present density-functional theory calculations for the geometry and conductance of 4,4-bipyridine (BPD) nanojunctions with $\mathrm{Au}$ and Pt electrodes. The fact that transport takes place via bipyridine's lowest unoccupied molecular orbital (LUMO) suggests that the Au-BPD junction should have larger conductance than the Pt-BPD junction due to the smaller work function of Au as compared to Pt. On the other hand, coupling to the local $d$ band is stronger in the case of Pt and this broadens the LUMO resonance. We find that these effects largely outbalance each other leading to conductances of $0.01 G_{0}$ and $0.02 G_{0}$ for the Au and Pt contacts, respectively $\left(G_{0}=2 e^{2} / h\right.$ is the conductance quantum). The effect of coupling to the electrodes is investigated by means of the group orbital which makes precise the concept of the local band. The construction allows us to explain and rationalize the first-principles results within a simple single-level model.
\end{abstract}

DOI: 10.1103/PhysRevB.78.165116

PACS number(s): 73.20.At, 73.40.Gk, 73.63.Rt

\section{INTRODUCTION}

Advances in the experimental techniques for manipulating and contacting individual molecules have made it possible to study charge transport at the single-molecule scale. Such experiments offer a unique opportunity to test and develop our understanding of quantum mechanics in open nanoscale systems, and might potentially pave the road for new technologies such as molecular electronics devices and high-precision chemical sensors. ${ }^{1-8}$ Alongside the development of experimental techniques, it has become possible to model the electrical properties of single molecules captured between metallic electrodes using first-principles methods. Due to the lack of detailed experimental control at the atomic scale, such calculations represent a fundamental tool for interpreting and rationalizing experimental data. ${ }^{9-14}$

The electrical conductance of bipyridine (BPD) molecules in a toluene solution dispersed between gold electrodes was measured in 2003 by Xu and Tao. ${ }^{15}$ The repeated and independent $\sim 1000$ measurement showed that the conductance was quantized in units of $0.01 G_{0}$ which was interpreted as the formation of stable contacts containing one or more molecules in parallel configurations. Several theoretical calculations on the gold-bipyridine junctions have been reported, and they consistently find that transport takes place via the lowest unoccupied molecular orbital (LUMO) of the bipyridine molecule. ${ }^{16-21}$ Furthermore, Stadler et al. ${ }^{18}$ studied the effect of the local contact geometry of Au-BPD junctions, and found that the conductance is entirely governed by the position of the LUMO with respect to the gold Fermi level which in turn is determined by the coordination number of the Au atom to which the molecule binds.

Apart from effects related to the local contact geometry, the electronic structure of the electrode should also influence the junction properties. First, the electrode work function determines the distance between the molecule's energy levels and the metal Fermi level when the molecule is far from the surface. Secondly, the fact that BPD binds at on-top metal sites implies that the BPD LUMO orbital (which has $\pi$ symmetry), will hybridize with the metal $d$ band, suggesting that the shape and position of the metal $d$ band should influence the electrical properties of the junction.

In this paper we present density-functional theory (DFT) calculations for the structure and conductance of molecular junctions consisting of bipyridine $\left(\mathrm{C}_{10} \mathrm{H}_{2} \mathrm{~N}_{2}\right)$ sandwiched between $\mathrm{Au}(111)$ and $\mathrm{Pt}(111)$ surfaces. The conductance is found to be rather similar for the two systems, namely $0.01 G_{0}$ and $0.02 G_{0}$ for the Au-BPD and Pt-BPD junctions, respectively. The current is carried exclusively via the BPD LUMO orbital and the conductance is determined by two effects: (i) the electrode work function and (ii) the position of the electrode $d$ band. The position of the LUMO in the Pt junction is shifted up by $0.4 \mathrm{eV}$ as compared to the LUMO in the $\mathrm{Au}$ junction due to the difference in work function of $\mathrm{Au}$ and Pt. On the other hand, the $d$ band lies higher in energy for $\mathrm{Pt}$ than for $\mathrm{Au}$, leading to stronger hybridization with the LUMO in the Pt-BPD. This effect broadens the LUMO resonance leading to an increase in conductance which counterbalances the opposite effect of the work function. A detailed picture of the effect of coupling to the metal band is obtained by constructing the group orbital of the LUMO. The group orbital makes precise the concept of the local band and provides a complete, quantitative description of the firstprinciples transmission function within a one-level junction model.

The paper is organized as follows. In Sec. II we briefly review the computational methods used in the current study and introduce the useful concept of the group orbital within the single-level Newns-Anderson model. In Sec. III we present the result for the calculated transport properties of the Pt-BPD-Pt and Au-BPD-Au junctions. Finally, Sec. IV contains a summary.

\section{METHOD}

Structural optimizations of the junction geometries were performed using the SIESTA DFT code. ${ }^{22}$ Troullier-Martin ${ }^{23}$ norm-conserving pseudopotentials are used to describe the 


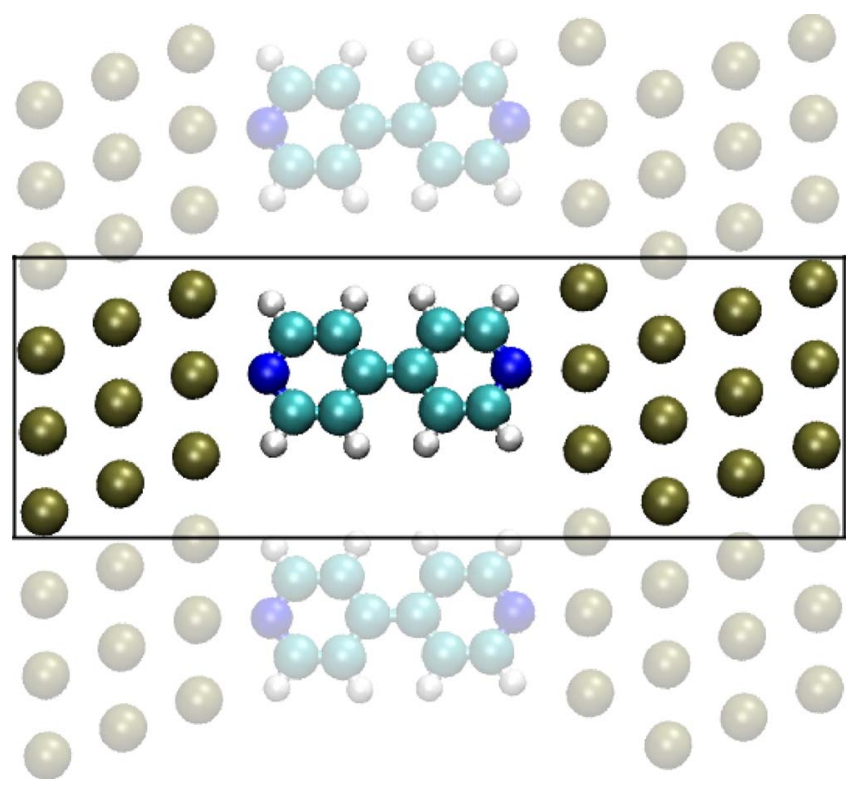

FIG. 1. (Color online) Supercell used for the metal-bipyridinemetal molecular junction.

ions and a double zeta polarized (DZP) basis has been used in all calculations. Exchange and correlation effects are included by the Perdew-Burke-Ernzerhof (PBE) (Ref. 24) functional. We use a mesh cutoff of 250 Ry for the density and an energy shift of 0.01 Ry for the basis functions.

The supercell used for the Au-BPD and Pt-BPD junctions is shown in Fig. 1. The scattering region consists of three and four atomic planes to the left and right of the molecule, respectively. Each plane contains $3 \times 3$ atoms and periodic boundary conditions are used in all directions. The metal atoms were kept fixed in their bulk configuration with lattice parameters $a=3.92 \AA$ for Pt and $a=4.08 \AA$ for Au. The electrode gap was varied in small steps and the atoms of the bipyridine molecule were relaxed at each step in order to determine the optimal electrode separation.

The conductance of the relaxed junctions was calculated using the nonequilibrium Green's function formalism with the electronic structure described by the SIESTA Hamiltonian. The starting point for the transport calculations is the general formula for the transmission function ${ }^{25,26}$

$$
T(\varepsilon)=\operatorname{Tr}\left[\Gamma_{R}(\varepsilon) G(\varepsilon) \Gamma_{L}(\varepsilon) G^{\dagger}(\varepsilon)\right],
$$

where $G(\varepsilon)$ denotes the retarded Green's function of the scattering region and $\Gamma_{L / R}(\varepsilon)$ describes the coupling to the left/ right lead. The linear response conductance can then be obtained by multiplying $T$ evaluated at the Fermi level by the conductance quantum, $G_{0}=2 e^{2} / h$. Due to the periodic boundary conditions in the plane perpendicular to the transport direction the transmission function is sampled over a $4 \times 4$ Monkhorst-Pack k-point grid in the Brillouin zone of the transverse plane. This is essential in order to avoid spurious features in the transmission function related to the quasi-one-dimensional structure of the lead when only a single $\mathbf{k}$ point is used.$^{27}$ In particular, we stress that with a proper k-point sampling, it is sufficient to include $3 \times 3$ metal atoms in the surface plane to obtain a fully converged transmission function. ${ }^{28}$ For more details of the transport method used here, we refer to Ref. 28.

\section{A. Newns-Anderson model and group orbital}

Taking Eq. (1) as a starting point, a simple but physically appealing model of electronic transport through a single level can be derived from the Newns-Anderson model ${ }^{29}$ together with the concept of a group orbital. In the NewnsAnderson model a single molecular orbital $|a\rangle$ with on-site energy $\varepsilon_{a}$ is coupled to left $(L)$ and right $(R)$ semi-infinite leads via the matrix elements $t_{\alpha \nu}=\langle a|\hat{H}| \alpha \nu\rangle$, where $\{|\alpha \nu\rangle\}$ denotes an orthonormal basis for lead $\alpha=L, R$. The retarded Green's function of the central site is given by

$$
G_{a}(\varepsilon)=\frac{1}{\varepsilon-\varepsilon_{a}-\Sigma_{L}(\varepsilon)-\Sigma_{R}(\varepsilon)},
$$

where the $\Sigma_{\alpha}$ is the self-energy of lead $\alpha$. The group orbital is defined as the orthogonal projection of the state $\hat{H}|a\rangle$ onto the lead states,

$$
\left|\gamma_{\alpha}\right\rangle=\frac{1}{V_{\alpha}} \sum_{\nu} t_{\alpha \nu}|\alpha \nu\rangle
$$

Here $V_{\alpha}=\left(\Sigma_{\nu}\left|t_{\alpha \nu}\right|^{2}\right)^{1 / 2}$ is a normalization constant. The group orbital for lead $\alpha$ is a weighted sum of lead states with weights given by the coupling matrix elements $t_{\alpha v}$. In real space it is therefore expected to be localized at the lead interface, near the contact atom. The group orbital has the key property that the coupling between the central site and any orbital in lead $\alpha$ orthogonal to $\left|\gamma_{\alpha}\right\rangle$ is zero. The self-energy then takes a particular simple form,

$$
\Sigma_{\alpha}=\left|V_{\alpha}\right|^{2} g_{\gamma \alpha}^{0},
$$

where $g_{\gamma \alpha}^{0}$ is the Green's function of the group orbital in lead $\alpha$ in the absence of coupling to the central site. The selfenergies can be split into real and imaginary parts $\Sigma_{\alpha}(\varepsilon)$ $=\Lambda_{\alpha}(\varepsilon)-i \Delta_{\alpha}(\varepsilon)$, where the imaginary part is given in terms of $V_{\alpha}, \varepsilon_{a}$, and the projected density of states (PDOS) $\rho_{\gamma \alpha}^{0}$ of the group orbital;

$$
\Delta_{\alpha}(\varepsilon)=\pi\left|V_{\alpha}\right|^{2} \rho_{\gamma \alpha}^{0} .
$$

The retarded self-energy in Eq. (4) is analytic in the upper half of the complex plane, and consequently the real part $\Lambda_{\alpha}(\varepsilon)$ is related to the imaginary part $\Delta_{\alpha}(\varepsilon)$ by a Hilbert transform. The coupling $\Gamma_{\alpha}$ entering Eq. (1) is given by

$$
\Gamma_{\alpha}(\varepsilon)=i\left[\Sigma_{\alpha}(\varepsilon)-\Sigma_{\alpha}^{\dagger}(\varepsilon)\right]=2 \Delta_{\alpha}(\varepsilon) .
$$

For symmetric coupling, $\Lambda_{L}=\Lambda_{R} \equiv \Lambda$ and $\Delta_{L}=\Delta_{R} \equiv \Delta$. The PDOS of $|a\rangle$ can now be written as

$$
\rho_{a}(\varepsilon)=\frac{2}{\pi} \frac{\Delta(\varepsilon)}{\left[\varepsilon-\varepsilon_{a}-2 \Lambda(\varepsilon)\right]^{2}+[2 \Delta(\varepsilon)]^{2}}
$$

and the transmission function of Eq. (1) takes the simple form

$$
T(\varepsilon)=\pi^{2}\left|V_{c}\right|^{2} \rho_{c}^{0}(\varepsilon) \rho_{a}(\varepsilon) .
$$


Expression (3) for the group orbital assumes that the lead basis functions $|\alpha \nu\rangle$ are orthogonal. As the atomic orbitals used by SIESTA are nonorthogonal, we perform a GramSchmidt orthonormalization starting from central orbital $|a\rangle$. The orthonormalization is conducted in the lead subspaces only and in practice only a finite number of lead basis functions near the molecule needs to be included. Having obtained the new orthonormal basis Eq. (3) can be applied to construct the group orbital.

\section{RESULTS}

Following the procedure described in Sec. II for relaxing the metal-BPD-metal junction geometries we found that BPD preferentially binds at an on-top site of the Pt(111) and $\mathrm{Au}(111)$ surfaces with little variation in the internal geometry of the molecule as a function of electrode separations. The minimum energy structure for the Pt junction was found for an electrode separation of $11.45 \AA$ where the following bond lengths were obtained: $d_{\mathrm{N}-\mathrm{Pt}}=2.19 \AA, d_{\mathrm{H}-\mathrm{C}}=1.09 \AA$, $d_{\mathrm{C}-\mathrm{N}}=1.35 \AA$, and $d_{\mathrm{C}-\mathrm{C}}=1.39 \AA$. By calculating the total energy of the system for different torsion angles, $\theta$, between the two pyridine rings, we found that the minimum energy configuration is achieved for $\theta=34^{\circ}$ and the energy maximum occurs for $\theta=90^{\circ}$. In addition the energy has a local maximum for the planar configuration, $\theta=0^{\circ}$. In units of $k_{B} T_{\mathrm{RT}}$ $\approx 25 \mathrm{meV}$, the energies of the extremal configurations are $E_{34^{\circ}}=0.0, E_{90^{\circ}}=5.0$, and $E_{0^{\circ}}=0.4$ with very similar values for the $\mathrm{Au}$ junction. The conductance is significantly more sensitive to the torsion angle and follows the law $G(\theta)$ $=G_{\max } \cos ^{2}(\theta)$ with $G_{\text {max }, \mathrm{Au}}=0.01 G_{0}$ and $G_{\mathrm{max}, \mathrm{Pt}}=0.02 G_{0}$ for the $\mathrm{Au}$ and $\mathrm{Pt}$ junctions, respectively. The cosine derives from the angular dependence of the overlap of the $\pi$ orbitals on the two pyridine rings as was also found for similar molecules in previous studies. ${ }^{30,31}$

Under room-temperature conditions, the thermal fluctuations of $\theta$ will be significant and the pyridine rings will not have a well-defined relative angle. Since we expect changes in the torsion angle to have a very similar effect on the $\mathrm{Au}$ and Pt junctions, and because we are mainly interested in comparing the two systems, we specialize for simplicity to the planar configuration in what follows.

For the Au-BPD-Au junction, we adopted the molecular geometry and $\mathrm{N}$-Au bond length from the relaxed Pt junction. Although the optimal N-Au bond length is slightly longer than the optimal N-Pt bond length $\left(d_{\mathrm{N}-\mathrm{Au}}=2.35 \AA\right)$ we use the same molecular geometries for the two systems for a more consistent comparison. In particular, this ensures that the observed differences are purely electronic in origin. ${ }^{32}$

In Fig. 2 we show the transmission functions for the AuBPD and Pt-BPD junctions. The inset of Fig. 2 is a zoom of the resonances in the region near the Fermi level. Both transmission resonances have Lorentzian-type shapes with almost identical widths. The Pt-BPD resonance is located $0.4 \mathrm{eV}$ above the Au-BPD resonance which is consistent with the fact that the work function of $\mathrm{Pt}(111)$ is $0.6 \mathrm{eV}$ larger than that of $\mathrm{Au}(111) .{ }^{33}$ This clearly suggests that the conductance of the Pt-BPD junction should be lower than the conductance of the Au-BPD junction. However, the calculated conduc-

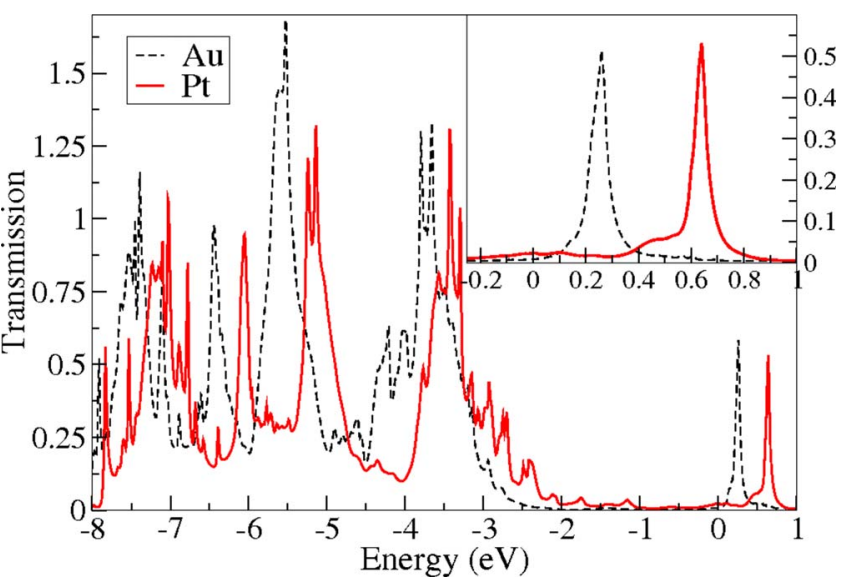

FIG. 2. (Color online) The transmission function for the Aubipyridine junction (dashed line) and the Pt-bipyridine junction (full line). The inset shows a close-up of the region around the Fermi level. In this region, the transmission function can be completely accounted for by the LUMO orbital of the bipyridine.

tance of the Pt-BPD contact $\left(0.02 G_{0}\right)$ is in fact two times larger than conductance of the Au-BPD contact $\left(0.01 G_{0}\right)$. From Fig. 2 this is clearly related to the distortion of the Pt-BPD resonance tail.

In order to investigate what causes the different shapes of the transmission resonances we start by identifying the molecular orbitals (MO) involved in the transmission resonances. To this end, we diagonalize the Hamiltonian within the molecular subspace, i.e., the space spanned by the atomic orbitals located at the BPD molecule, to obtain a "rotated" and equivalent basis of MO. We then consider transport through each of the MO separately by calculating the transmission function using a single MO to describe the BPD molecule. (We have checked that we reproduce the result of Fig. 2 when all MO are included in the calculation.) In this way we find that the transmission function in the relevant energy window around the Fermi level can be completely accounted for by the LUMO orbital. The energies for the uncoupled LUMO orbitals, i.e., the eigenvalues obtained from diagonalizing the Hamiltonian within the molecular subspace, are found to be $0.51 \mathrm{eV}$ and $0.19 \mathrm{eV}$ for the PtBPD and Au-BPD junctions, respectively.

In Fig. 3 we show isosurfaces of the LUMO in the PtBPD junction and the corresponding group orbitals constructed as described in Sec. II A. As expected the group orbitals are localized at the molecule-electrode interfaces and their symmetry follows from the $\pi$ symmetry of the LUMO.

The PDOS of the group orbitals, $\rho_{\gamma}^{0}$, are shown in Fig. 4 (thin red line) for the Pt-BPD and Au-BPD junctions. For comparison we also show the PDOS for the $d$ orbital of the contacting metal atom which has the largest weight in the expansion of the group orbital (black dashed line). From the similarity of the two densities we infer that for all practical purposes the group orbital can be replaced by an atomic $d$ orbital. The group orbital PDOS, or local $d$ band, can be approximated by a semielliptical band (thick black line) plus a constant background representing the contributions to the group orbital from the wide metal $s$ band. The coupling between the LUMO and the group orbital as well as the (un- 


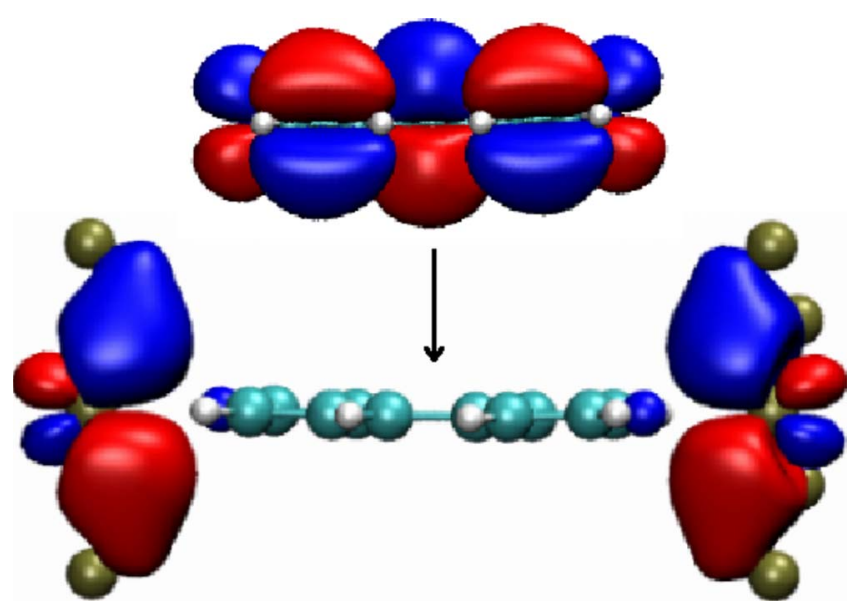

FIG. 3. (Color online) Isosurfaces of the LUMO (above) and the corresponding group orbitals (below) for the Pt-BPD junction. The group orbitals are localized at the molecule-electrode interface, and their symmetry follows from the $\pi$ symmetry of the LUMO.

coupled) LUMO on-site energy, $\varepsilon_{a}$, can be directly obtained from the DFT calculation. Finally, the Hilbert transform of the model semielliptical band can be performed to obtain the full self-energies, $\Sigma_{L / R}$, and the PDOS of the LUMO can be constructed from Eq. (7). The result, shown by the blue line in Fig. 4, reproduces the resonances from the full DFT calculation very closely. In particular it is clear that the long tail of the LUMO resonance in Pt-BPD junction is due to hybridization with the $\mathrm{Pt} d$ band. On the other hand hybridization effects do not affect the LUMO resonance in the Au-BPD junction because the filled $\mathrm{Au} d$ band lies too far away from the LUMO.

\section{SUMMARY}

With the aim of investigating the role of the electrodes for transport in molecular junction we have performed DFT calculations for the structure and conductance of 4,4-bipyridine molecules suspended between $\mathrm{Au}$ and $\mathrm{Pt}$ surfaces, respectively. Transport takes place via the LUMO orbital and the conductance is determined by two effects: (i) the metal work function which determines the position of the (uncoupled) LUMO with respect to the metal Fermi level, and (ii) the energetic position of the metal $d$ band which controls the coupling strength. In the case of $\mathrm{Au}$ and $\mathrm{Pt}$ the two effects are significant but largely outbalance each other leading to
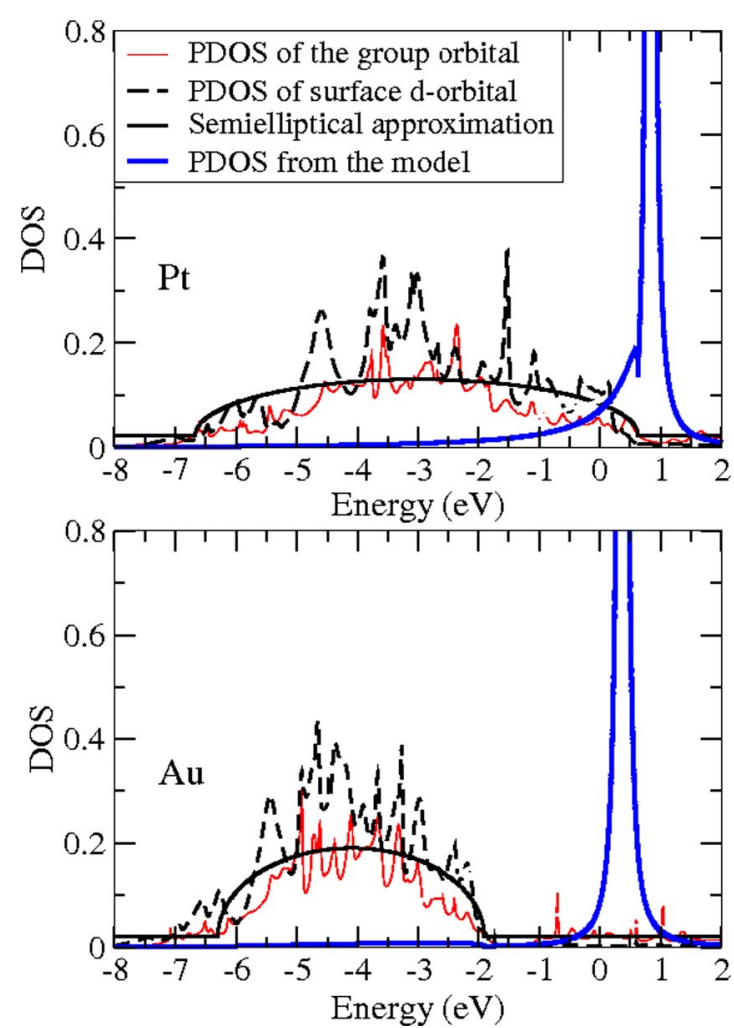

FIG. 4. (Color online) Projected density of states for the group orbital of the LUMO (full red line) and an atomic $d$ orbital of the contacting Au atom (black dashed line). Also shown is a semielliptical approximation to the group orbital PDOS (full black line) and the resulting PDOS of the LUMO (full blue line) as calculated from Eq. (7) when the semielliptical band is used together with the coupling strength, $V$, and level position $\varepsilon_{a}$ extracted from the DFT calculation. Notice that the distortion of the LUMO resonance tail in the case of Pt-BPD junction is due to the Pt $d$ band.

rather similar conductances for the two systems. Finally, it was shown how the so-called group orbital can be constructed from the DFT calculation and how it provides a simple yet quantitative model for the metal-molecule hybridization.

\section{ACKNOWLEDGMENTS}

The authors acknowledge support from The Lundbeck Foundation's Center for Atomic-scale Materials Design (CAMD) and from the Danish Center for Scientific Computing through Grant No. HDW-1103-06.
${ }^{1}$ H. Ohnishi, Y. Kondo, and K. Takayanagi, Nature (London) 395, 780 (1998).

${ }^{2}$ J. Joachim, J. K. Gimzewski, and A. Aviram, Nature (London) 408, 541 (2000).

${ }^{3}$ R. H. M. Smit, Y. Noat, C. Untiedt, N. D. Lang, M. C. van Hemert, and J. M. van Ruitenbeek, Nature (London) 419, 906 (2002).

${ }^{4}$ M. A. Reed, C. Zhou, C. J. Muller, T. P. Burgin, and J. M. Tour,
Science 278, 252 (1997)

${ }^{5}$ D. M. Eigler, C. P. Lutz, and W. E. Rudge, Nature (London) 352, 600 (1991).

${ }^{6}$ A. Aviram and M. A. Ratner, Chem. Phys. Lett. 29, 277 (1974).

${ }^{7}$ J. Reichert, R. Ochs, D. Beckmann, H. B. Weber, M. Mayor, and

H. v. Löhneysen, Phys. Rev. Lett. 88, 176804 (2002).

${ }^{8}$ K. Balasubramanian and M. Burghard, Small 1, 180 (2005).

${ }^{9}$ A. Nitzan and M. A. Ratner, Science 300, 1384 (2003). 
${ }^{10}$ J. Taylor, H. Guo, and J. Wang, Phys. Rev. B 63, 245407 (2001).

${ }^{11}$ Y. Xue, S. Datta, and M. A. Ratner, Chem. Phys. 281, 151 (2002).

${ }^{12}$ M. Brandbyge, J. L. Mozos, P. Ordejón, J. Taylor, and K. Stokbro, Phys. Rev. B 65, 165401 (2002).

${ }^{13}$ S. Y. Quek, L. Venkataraman, H. J. Choi, S. G. Louie, M. S. Hybertsen, and J. B. Neaton, Nano Lett. 7, 3477 (2007).

${ }^{14}$ D. Djukic, K. S. Thygesen, C. Untiedt, R. H. M. Smit, K. W. Jacobsen, and J. M. van Ruitenbeek, Phys. Rev. B 71, 161402(R) (2005).

${ }^{15}$ B. Xu and N. J. Tao, Science 301, 1221 (2003).

${ }^{16}$ T. Tada, M. Kondo, and K. Yoshizawa, J. Chem. Phys. 121, 8050 (2004).

${ }^{17}$ S. Hou, J. Zhang, R. Li, J. Ning, R. Han, Z. Shen, X. Zhao, Z. Xue, and Q. Wu, Nanotechnology 16, 239 (2005).

${ }^{18}$ R. Stadler, K. S. Thygesen, and K. W. Jacobsen, Phys. Rev. B 72, 241401(R) (2005).

${ }^{19}$ R. Stadler and K. W. Jacobsen, Phys. Rev. B 74, 161405(R) (2006).

${ }^{20}$ A. J. Perez-Jimenez, J. Phys. Chem. B 109, 10052 (2005).

${ }^{21} \mathrm{X}$. Wu, Q. Li, J. Huang, and J. Yang, J. Chem. Phys. 123, 184712 (2005).

${ }^{22}$ J. M. Soler, E. Artacho, J. D. Gale, A. Garcia, J. Junquera, P.
Ordejón, and D. Sanchez-Portal, J. Phys.: Condens. Matter 14, 2745 (2002).

${ }^{23}$ N. Troullier and J. L. Martins, Solid State Commun. 74, 613 (1990).

${ }^{24}$ J. P. Perdew, K. Burke, and M. Ernzerhof, Phys. Rev. Lett. 77, 3865 (1996).

${ }^{25}$ Y. Meir and N. S. Wingreen, Phys. Rev. Lett. 68, 2512 (1992).

${ }^{26}$ K. S. Thygesen, Phys. Rev. B 73, 035309 (2006).

${ }^{27}$ K. S. Thygesen and K. W. Jacobsen, Phys. Rev. B 72, 033401 (2005).

${ }^{28}$ M. Strange, I. S. Kristensen, K. S. Thygesen, and K. W. Jacobsen, J. Chem. Phys. 128, 114714 (2008).

${ }^{29}$ D. M. Newns, Phys. Rev. 178, 1123 (1969).

${ }^{30}$ F. Pauly, J. K. Viljas, J. C. Cuevas, and Gerd Schön, Phys. Rev. B 77, 155312 (2008).

${ }^{31}$ L. Venkataraman, J. E. Klare, C. Nuckolls, M. S. Hybertsen, and M. L. Steigerwald, Nature (London) 442, 904 (2006).

${ }^{32}$ The conductance of the optimized, planar Au-BPD-Au junction is slightly lower than the conductance obtained when the $\mathrm{N}-\mathrm{Au}$ bond length is fixed to the N-Pt value, namely $0.007 G_{0}$ (Ref. 18) as compared to $0.01 G_{0}$.

${ }^{33}$ P. C. Rusu and G. Brocks, Phys. Rev. B 74, 073414 (2006). 